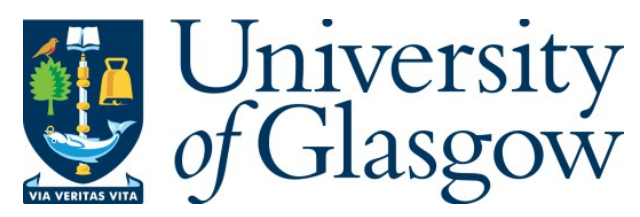

Gomes, J., Salgueiro, P., Inácio, J., Amaro, A., Pinto, J., Tait, A., Shiels, B., Pereira da Fonseca, I., Santos-Gomes, G., and Weir, W. (2016) Population diversity of Theileria annulata in Portugal. Infection, Genetics and Evolution, 42, pp. 14-19.

There may be differences between this version and the published version. You are advised to consult the publisher's version if you wish to cite from it.

$\underline{\text { http://eprints.gla.ac.uk/120663/ }}$

Deposited on: 04 August 2016

Enlighten - Research publications by members of the University of Glasgow http://eprints.gla.ac.uk 


\title{
Population diversity of Theileria annulata in Portugal
}

Jacinto Gomes ${ }^{\mathrm{a}, \mathrm{b},{ }^{*},}$, Patrícia Salgueiro ${ }^{c}$, João Inácio ${ }^{\mathrm{c}, \mathrm{d}}$, Ana Amaro ${ }^{\mathrm{a}}$, João Pinto ${ }^{c}$, Andy Tait ${ }^{\mathrm{e}}$, Brian Shiels ${ }^{\mathrm{e}}$, Isabel Pereira da Fonseca ${ }^{\mathrm{b}}$, Gabriela Santos-Gomes ${ }^{\mathrm{c}}$, William Weir ${ }^{\mathrm{e}}$

a Unidade Estratégica de Investigação e Serviços em Produção e Saúde Animal, Instituto Nacional de Investigação Agrária e Veterinária, I.P., Lisbon, Portugal

${ }^{\text {b }}$ Centro de Investigação Interdisciplinar em Sanidade Animal, Faculdade de Medicina Veterinária, Universidade de Lisboa, Lisbon, Portugal

${ }^{c}$ Global Health and Tropical Medicine Centre (GHTM), Instituto de Higiene e Medicina Tropical, Universidade Nova de Lisboa, Lisbon, Portugal

d School of Pharmacy and Biomolecular Sciences, University of Brighton, Brighton, United Kingdom

e Institute of Biodiversity, Animal Health and Comparative Medicine, College of Medical, Veterinary and Life Sciences, University of Glasgow, Glasgow, United Kingdom

*Corresponding author at: Instituto Nacional de Investigação Agrária e Veterinária, I.P. (INIAV, I.P.), E-mail address: jacinto.gomes@iniav.pt

\begin{abstract}
The tick-borne protozoan parasite Theileria annulata causes tropical or Mediterranean theileriosis, a severe disease of cattle that occurs across the Mediterranean littoral, the Middle East and Southern Asia. In the Mediterranean region, the disease has long been perceived as being a constraint to livestock production in North Africa and Turkey but was believed to have minimal impact in Southern European countries. It has recently been demonstrated that in Southern Portugal, which contains half the bovine population in the country, the prevalence of $T$. annulata is approximately $30 \%$. While the population genetics of the parasite and the multiplicity of infection in the bovine host have been studied in a number of countries, no information is currently available on the composition of the parasite population in Southern Europe or its relationship to populations in bordering regions. In addition, since no vaccines or chemoprophylactic drugs against theileriosis have been employed in Portugal, it can be predicted that local parasite genetic diversity has not been influenced by such factors. A parasite genotyping system based on micro- and mini-satellite


loci amplification was used to perform the first genetic analysis of $T$. annulata populations in Portugal. DNA was extracted from blood samples $(n=90)$ collected from $T$. annulata infected cattle in twelve farms within four regions of Southern Portugal. A diversity of genotypes and a high multiplicity of infection were found, suggesting that the parasite possesses a panmictic population in this region, similar to countries where tropical theileriosis has long been recognized. In comparison with genotypes found in Tunisia and Turkey, parasites from Portugal are genetically distinct and show lower genetic diversity, although infected cattle display a similar multiplicity of infection. This study provides valuable information about T. annulata population genetics, potentially allowing a more rational design and application of control measures.

Keywords: Theileria annulata, population genetics, micro-satellites, mini-satellites, multiplicity of infection,

\section{Introduction}

Theileria annulata is a tick-borne parasite that causes tropical or Mediterranean theileriosis, an economically important disease of cattle that occurs around the Mediterranean region, the Middle East and Southern Asia (Gubbels et al., 1999; García-Sanmartín et al., 2006). The parasite is transmitted by two- and three-host ticks of the genus Hyalomma (Ghosh et al., 2008; Gharbi et al., 2013). Theileria annulata sporozoites enter the bovine host during tick feeding and rapidly invade cells of, primarily, myeloid lineage. After several rounds of schizogony linked to host cell division, merozoites are produced, released upon host cell destruction and subsequently invade erythrocytes (Dobbelaere and Heussler, 1999). Piroplasm-infected erythrocytes are ingested by ticks where the sexual phase occurs, followed by the production of thousands of infective haploid sporozoites in the salivary glands. The parasite has a primarily haploid life-cycle with a brief diploid phase in the tick corresponding to the zygote and kinete stages (Mehlhorn and Schein, 1984).

In Portugal, the importance of theileriosis is becoming increasingly recognized (Branco et al., 2010; Silva et al., 2010) and a recent epidemiological study determined an increased prevalence in the south of the country. In a cross-sectional study in Southern Portugal, around $30 \%$ of cattle were found to be infected within an area that represents around $50 \%$ of this country's cattle population (Gomes et al., 2013). The majority of reported clinical cases occur in young calves or imported animals, and a state of endemic stability is presumed to occur. In the northern part of the country, where infected animals and vector ticks are also known to co-exist, the disease is less common and tick challenge is presumed to be relatively lower. Animals that survive acute disease become carriers of $T$. annulata and 
play an important role as reservoirs for the maintenance of the parasite population (D'Oliveira et al., 1995). Vaccination with a live attenuated vaccine and chemoprophylaxis with buparvaquone are available in certain countries. However, neither of these control measures is available in Portugal. The only available intervention is the use of acaricides for tick control.

A number of studies have shown considerable levels of genetic diversity in the T. annulata population using a variety of serological and molecular approaches (Ben Miled et al., 1994, Shiels et al., 1986, Gubbels et al., 2000, Schnittger et al., 2002, Weir et al., 2010). Population genetic studies of $T$. annulata have previously been performed using a panel of micro- and mini-satellite markers (Weir et al., 2007). This genotyping approach has also been applied to Apicomplexan species such as Plasmodium falciparum (Anderson et al., 2000) and to T. parva, a phylogenetically close relative of T. annulata (Oura et al., 2005; Muleya et al., 2012). A study within Tunisian and Turkish T. annulata populations demonstrated that genetic differentiation could be detected between geographically separated populations (Weir et al., 2007). Although a moderate level of differentiation of populations between countries was shown, complete genetic isolation between Tunisia and Turkey was not observed. The level of genetic differentiation within Turkish sub-populations was comparable to that observed within Tunisia sub-populations with both countries evidencing linkage disequilibrium and some geographical sub-structuring.

The present study aims to investigate the genetic diversity of $T$. annulata in Portugal and perform a comparative analysis with the data available from Tunisia and Turkey. We analyzed samples from twelve farms with infected cattle from four distinct regions of southern Portugal. To investigate the structure of parasite population in relation with the different geographical regions and to evaluate the multiplicity of infection in the cattle host, we used a panel of micro- and mini-satellites. Such information may provide important baseline data upon which suitable control policies for this parasite may be developed in the future.

\section{Materials and methods}

\subsection{Sampling and DNA extraction}

Bovine blood samples were collected into EDTA tubes between January and June 2012 from several farms in southern Portugal. All samples were taken from animals older than six months and without clinical signs of theileriosis. A total of $800 \mu \mathrm{l}$ of whole blood was used for DNA extraction. Red cells were lysed using distilled water and centrifuged at $4,500 \mathrm{~g}$ (2 cycles of 5 minutes). The supernatant was discarded and the remaining pellet reconstituted with $200 \mu \mathrm{l}$ of water. DNA was extracted using the High Pure PCR Template Preparation Kit (Roche, Switzerland) according to the manufacturer's instructions. Samples were tested for 
the presence of $T$. annulata DNA using real-time PCR (Santos et al., 2013). Ninety samples, collected from bovines from twelve farms within four regions, yielding positive results for the presence of $T$. annulata were selected for genotyping. The Ankara (D7) clone (Shiels et al., 1992) was used as a parasite-positive control sample. DNAse free water (GIBCO) was used as a negative control.

\subsection{Genotyping and allele calling}

Genotyping of DNA samples was performed by PCR amplification of four previously described micro-satellites (TS5, TS9, TS12 and TS16) and five mini-satellite markers (TS6, TS8, TS15, TS20 and TS25) under the conditions described by Weir et al. (2007). The amplified products were separated on a capillaryautomated sequencer (ABI 3100 Genetic Analyser). DNA fragment size was determined by comparison with a set of ROX-labelled size standards (GS500 markers, ABI). The electrophoretograms obtained for each sample were analysed using the Peak Scanner Software v1.0 (ABI) in order to identify and quantify peaks representing amplicons. Only peaks with an area greater than $32 \%$ of the largest peak area were recorded. Traces were manually inspected and artefactual peaks, considered to be due to either a stutter or an untemplated 'A' effect, were eliminated. For the construction of multilocus genotypes (MLG) for each sample, we identified the predominant peak (predominant allele) at each locus in each sample and assigned it to a bin. The genotyping of a cloned parasite isolate (Ankara D7) in this study allowed the MLG dataset to be harmonised with and compare to a previous Tunisian and Turkish dataset (Weir et al., 2011).

\subsection{Data analysis}

Allelic frequencies and expected (gene diversity) heterozygosity were calculated using the Excel Microsatellite Toolkit (Park, 2001). Unbiased allelic richness was calculated using HPRARE 1.0 using the rarefaction statistic (Kalinowski, 2005). As T. annulata is haploid during the blood stages, the Multiplicity of Infection (MOI) can be determined by the number of different alleles detected within each sample. Since multiple loci were genotyped, the mean number of alleles per isolate was used as a proxy-estimate of $\mathrm{MOI}$ for comparisons between regions of Portugal and countries and differences were tested by ANOVA.

Population differentiation analysis was performed with MLG data. Estimates of Wright fixation index $\left(F_{S T}\right)$ were made according to Weir and Cockerham (1984) with the software ARLEQUIN (Excoffier et al., 2005), to determine genetic differentiation between samples and the respective statistical significance. A value of 0 for $F_{S T}$ indicates no differentiation, values between 0 and 0.05 are indicative of little genetic differentiation, values between 0.05 and 0.15 are indicative of moderate differentiation, values between 0.15 and 0.25 are indicative of 
great differentiation and values above 0.25 show very great genetic differentiation (Balloux and Lugon-Moulin, 2002). For multiple tests, the significance level was corrected according to the sequential Bonferroni procedure to avoid type I errors (Holm, 1979).

The LIAN linkage analysis software, Version 3.6 (http://guanine.evolbio.mpg.de/cgibin/lian/lian.cgi.pl) was used to test the null hypothesis of Linkage Equilibrium (LE) in all four regions in Portugal and among Portugal, Tunisia and Turkey using the standard index of association $\left(\mathrm{I}_{\mathrm{A}}^{\mathrm{S}}\right)$ (Haubold and Hudson 2000), a measure of linkage equilibrium/disequilibrium. This software tests for independent assortment of alleles by determining the number of loci that differs at each pair of MLG. Variance $\left(V_{D}\right)$ is calculated from the distribution of mismatch values and is compared with the expected variance $\left(V_{e}\right)$ for LE. The null hypothesis of LE is tested $\left(V_{D}=V_{e}\right)$ by a Monte Carlo simulation or a parametric method and the results provide $95 \%$ confidence limits, respectively designated as $L_{M C}$ and $L_{\text {PARA. If }} V_{D}$ is greater than $L$ and a positive value of the index of association is obtained, the null hypothesis is discarded and $L D$ is indicated. The higher the $\mathrm{I}_{\mathrm{A}}^{\mathrm{S}}$ value, the greater the level of linkage disequilibrium, while a value close to zero indicates LE.

A factorial correspondence analysis (FCA) was also performed on MLG data from Portugal and comparing the countries (Portugal, Tunisia and Turkey), using the software GENETIX 4:05 (Belkhir et al. 2004). This form of multivariate analysis allows underlying trends among parasite genotypes to be investigated with MLGs being graphically represented by points in bi- or tri-dimensional space.

Bayesian clustering analysis with STRUCTURE 2.3.4 (Pritchard et al. 2000) was performed for the Portuguese samples and also for a set of parasite samples from Portugal, Tunisia and Turkey. In both cases a 'no admixture' model was used without prior information on sampling groups, assuming correlated allele frequencies among populations ( $\lambda$ was set at 1 ). Ten independent runs with $10^{5}$ burn-in steps and $20^{5}$ iterations were done for each value of $K(K$ varied from 1 to 5 in $A$, and from 1 to 4 in $B)$. The number of genetic clusters $(K)$ was inferred with the software STRUCTURE HARVESTER (Earl and von Holdt 2011). The information from the outputs of each $K$ (10 runs) was compiled by the Greedy method implemented in CLUMPP (Jakobsson and Rosenberg 2007).

The possible relationship between genetic differentiation (pairwise $F_{\mathrm{ST}}$ calculated in ARLEQUIN) and geographical distances (in $\mathrm{km}$ ) among regions was assessed using Mantel tests. These were carried out with the program IBDWS 3.23 (Jensen et al. 2005) with 10,000 randomizations. 


\section{Results}

\subsection{Allelic diversity and multiplicity of infection}

Theileria annulata positive samples were selected for genotyping, comprising 40 samples from Évora region (four farms), 29 samples from Portalegre region (five farms), twelve samples from Ribatejo region (two farms) and nine samples from Beja (one farm).

The number of alleles per marker within a single sample ranged from one to ten depending on the locus (Table 1) and the mean number of alleles per locus per sample ranged from 1.62 (for TS25) to 4.40 (for TS8). The markers TS16 and TS25 showed a maximum of four alleles while TS8 and TS20 showed no more than ten alleles per isolate. The comparison of the frequencies of the predominant alleles was performed as shown for marker TS5 (Figure 1). Descriptive statistics for MOI calculated across each of the nine micro- and minisatellite loci showed that every isolate represented a mixed infection, with several alleles identified at one or more loci. To provide a measure of the $\mathrm{MOI}$ within each isolate, the mean number of alleles across all loci was calculated for each isolate for each of the four regions (Table 2). Portuguese isolates had a mean of $3.06( \pm 0.75)$ alleles per locus and a range between 1.11 and 4.50 alleles. $\mathrm{MOI}$ values estimated for the four regions were similar, showing no statistically significant differences between the two regions with greatest number of farms and samples (Évora and Portalegre). The samples collected in these two regions presented a mean number of alleles per locus per isolate of 3.16 and 2.83 , respectively. The only significant difference was found between Portalegre farms and the only farm evaluated in Beja region $(p<0.05)$.

Comparing the three countries, the Turkish isolates had a mean of 3.12 and the Tunisian isolates had a mean of 2.54 alleles. MOI values of Portugal and Tunisia showed significant differences $(p<0.001)$ however, no difference was obtained between Portugal and Turkey (Table 2).

\subsection{Population diversity and geographical sub-structuring}

A MLG was constructed for each isolate based on the predominant allele at each locus. A total of 90 different genotypes were obtained and a descriptive summary of this data is presented in Table 3. A total of 97 alleles in all loci were found in the Portuguese population compared with 177 in Tunisia and 272 in Turkey. Comparing the unbiased allele richness, which takes into account sampling size disparity, the Portuguese population showed 102 alleles in all loci compared to the 181 in Tunisia and 199 in Turkey. Many of the alleles are common to all three populations, such as those identified using markers TS9 and TS25 with no private alleles detected in the population from Portugal, while other markers showed population-specific alleles, such as TS20, which had nine private alleles in Portugal. 
Compared with the Turkish population, which had 112 private alleles over all loci, Portugal has a lower number of 31 , similar to the Tunisia population, which has 33 private alleles.

Expected heterozygosity across all loci in Portugal was 0.625 , considerably lower than in Turkey (0.872) and Tunisia (0.884) (Table 4).

Genetic differentiation values $\left(\mathrm{F}_{\mathrm{ST}}\right)$ obtained indicates a moderate degree of differentiation between Portugal and Tunisia (0.069), a low degree of differentiation between Portugal and Turkey (0.047) and the lowest $F_{S T}$ was obtained in the comparison between Turkey and Tunisia (0.016) (Table 5). A low and non-significant degree of differentiation was detected among all four Portuguese regions. A lower level of parasite genetic diversity, as measured by expected heterozygosity, was observed within Portugal compared to the other two countries. No significant correlation was found between genetic and geographic distances. These levels of genetic differentiation were supported by both results of the FCA (Figure 2) and Bayesian clustering analysis.

The FCA for the Portuguese isolates was unable to separate isolates from the four regions of Portugal (Figure 2a), although differing patterns for the four regions could be discerned. It was observed that the majority of the Évora isolates clustered closely and these are largely separated from the Ribatejo isolates on the first axis of the FCA. Similarly, Portalegre and Beja isolates can largely be distinguished on the second axis. These results suggested that there is a degree of geographical sub-structuring. The FCA for the three countries (Figure 2b) evidenced a complete separation of populations, however the Tunisian and Turkish populations were more closely related while the Portuguese population was more distant, thus corroborating the geographical sub-structuring indicated with the genetic analysis.

The Bayesian analysis implemented in STRUCTURE revealed a homogeneous population in Portugal $(K=1)$. From analysis of the populations across the three countries, Portugal, Tunisia and Turkey, the best $K$ value was 2 , separating Portugal from a cluster grouping Tunisia and Turkey.

\subsection{Linkage analysis}

The standard index of association $\left(\mathrm{I}_{\mathrm{A}}^{\mathrm{S}}\right)$ was calculated using the MLG dataset to determine whether an association could be found between alleles when all pair-wise combinations of loci were examined. The statistical analysis of $\mathrm{I}_{\mathrm{A}}^{\mathrm{S}}$ values allowed the null hypothesis of random mating to be tested and to investigate whether the high levels of diversity observed in these populations could be explained by frequent genetic exchange.

The LD analysis was performed combining data for populations from the three countries, pair-wise and separately. The combined data showed an $\mathrm{I}_{\mathrm{A}}^{\mathrm{S}}$ value of 0.054 , while the pair- 
wise combination of data representing Portugal and Tunisia had a higher value of 0.168 and for Portugal and Turkey it was 0.061 , three times higher than the value computed for Tunisia and Turkey (0.0201). Linkage analysis was performed for each geographically defined population at the regional (Table 6) and farm level. The null hypothesis of linkage equilibrium could not be rejected in two regions with the smaller sample sizes, Ribatejo and Beja, while LD was observed in the other two regions. In those regions where LD was observed (Évora and Portalegre), when each farm was treated as a single population, LD was no longer detectable within three of the four farms at Évora and within three of the five farms from Portalegre (one farm was excluded because only three animals were evaluated). The isolates from the LD farm of Évora and Portalegre had an $\mathrm{I}_{\mathrm{A}}^{\mathrm{S}}$ of 0.092 and 0.143 , respectively. These values indicate a non-random association of alleles, evidencing particular combinations of alleles from different loci found more frequently than would be expected on the basis of random mating. When linkage analysis was performed using three farms in Évora with LE, LD was maintained with an $\mathrm{I}_{\mathrm{A}}^{\mathrm{S}}$ value of 0.019. The same occurred in Portalegre where, after removing the genotypes from the farm in LD from analysis, LD was still maintained $\left(I_{A}^{S}=0.0216\right)$.

\section{Discussion}

The present study represents the first broad population genetic analysis of $T$. annulata in Portugal. Establishment of control strategies should take into account the potential diversity of parasite populations and therefore it is important that genetic variation is investigated and quantified. Analysis of Portuguese isolates of $T$. annulata demonstrates that, similar to other endemic countries, the parasite population is highly diverse. In almost every animal positive for T. annulata a high MOI was observed. Similar MOI levels were observed in Turkey and lower values were recorded in Tunisia (Weir et al., 2007; Weir et al., 2011). MOI is an epidemiological measure of the number of parasite genotypes per infected individual, frequently used in malaria research, which can improve knowledge of parasite populations in aspects such as the dynamics of infection and as an indicator of immune status, pathogenesis, or the effect of transmission intensity and drug efficacy (Ross, 2012). The differences in $\mathrm{MOI}$ found between countries may reflect subtle differences in epidemiology such as transmission intensity, which may be related to variation in the level of cattle infestation with ticks but also with the prevalence of $T$. annulata infection in the tick population. In Portugal, there are two tick species, Hyalomma lusitanicum and H. marginatum (Estrada-Peña and Santos-Silva, 2005) that can be infected by $T$. annulata and either may have different vector competence from the other species that occur in Turkey and Tunisia. Other tick species are present in Tunisia, with Hyalomma scupense (syn. H. detritum) being one of the most common and economically important species found on 
livestock, particularly cattle. This species is the major vector of T. annulata (Bouattour, 1996) but other species like $H$. marginatum and $H$. anatolicum are also found in this country. In Turkey there are four Hyalomma species responsible for the transmission of $T$. annulata (Aktas et al., 2004).

Since the herds in the studied regions in Portugal have a low number of clinical cases and these occur mainly in young calves and introduced non-immunized adults, we can consider these areas of endemic stability. Due to the similar epidemiology of tropical theileriosis in different regions of Portugal, in terms of prevalence and disease presentation, only limited variation of $\mathrm{MOI}$ among Portuguese farms and regions was anticipated. Host age was previously demonstrated to be a significant determinant of $\mathrm{MOI}$ in Turkey and Tunisia because of the increased tick exposure over time and multiple re-infections of animals during the tick season (Weir et al., 2011). The number of co-infecting genotypes gradually increases over time, presumably as the animal is exposed to new genotypes. In contrasts to the previous study, which included a significant number of calves (Weir et al., 2011), the focus of sampling in the present study was on adult carrier animals and therefore the relationship between host age and $\mathrm{MOI}$ could not be explored. No association of $\mathrm{MOI}$ was detected with different cattle breeds, although the number of animals representing each breed was relatively low and therefore there was little statistical power to investigate this issue. Further studies are required to determine if this metric can be related to variation in breed susceptibility and disease tolerance.

Some degree of genetic differentiation between geographically separated populations of T. annulata was revealed in our study. A moderate level of differentiation was detected between Portugal and Tunisia and between Portugal and Turkey. A degree of genetic differentiation between $T$. annulata populations in Tunisia and Turkey was also previously detected by Weir et al. (2011). Similarly to other studies, the level of differentiation between regions within a single country is lower than between countries. This agrees with the observation that no major geographical barriers exist between regions of Portugal and there is documented cattle movement between regions for commercial reasons. The differentiation observed between populations in different countries is likely to be the consequence of genetic isolation, consistent with geographical and trade barriers hampering the free flow of parasite genetic material. Nevertheless, complete genetic isolation between the three countries was not observed, which may be due to: (a) parasite populations having become isolated relatively recently; or (b) occurrence of cattle movement between neighboring countries, allowing a limited level of parasite gene flow across the whole region. This may be the case in Tunisia and Turkey, as the disease is present in the intervening countries of Libya, Egypt and Israel. In contrast, Portugal is geographically distant from Tunisia and Turkey and there is no documented cattle movement between Portugal and these countries. 
Significant LD detected in populations of the three countries was consistent with genetic differentiation and geographical sub-structuring between Portugal and the other populations. Although the index of association across the four regions of Portugal was low (0.0272) it was slightly higher than in Turkey and Tunisia. The regions of Beja, with only one studied farm, and of Ribatejo, with two farms evaluated, revealed LE when analyzed independently. The regions with larger sample sizes and with more farms (Portalegre and Évora) were in slight LD. When analyzing within farms, LD was identified only in one farm from each region. When those farms are removed from the analysis, Portalegre and Évora sub-populations were still in LD. This mild LD results within two farms cannot be explained by geographical substructuring. While it is possible that factors such as influx of new genotypes (Wahlund effect) or an epidemic population structure may underlie this result, it must be borne in mind that the LD observed is very limited and may be introduced by particular genotypes being slightly over-sampled in the course of the study for a variety of reasons. Recombination, as measured by genetic linkage, in the Portuguese $T$. annulata is undoubtedly frequent and is at a broadly similar level to that observed in Tunisia. Although animals are kept exclusively on farms and are not free-roaming, it is known that there are movements of cattle from farm to farm particularly in neighboring regions (Batista et al., 2007). Our data suggests that cattle movement is insufficient to homogenise the present parasite population and that geographical sub-structuring is maintained at the regional level. The parasite population is essentially panmictic with only a minor disturbance showed by the low levels of $\mathrm{I}_{A}^{S}$ and only a limited level of LD.

In summary, while Portugal has not been perceived to experience the same problem of tropical theileriosis in comparison to other Mediterranean countries, it is clear that the extent of genetic diversity of $T$. annulata is not wholly dissimilar from countries where the disease has long been accepted as one the principal constraints to cattle production.

\section{Acknowledgements}

This work was partially supported by CIISA (UID/CVT/00276/2013). P. Salgueiro was supported by Fundação para a Ciência e a Tecnologia (FCT), through the grant SFRH/BPD/72532/2010. 
Table 1. Theileria annulata allelic diversity per sample per locus in Portugal

\begin{tabular}{lccccccccc}
\hline & TS5 & TS6 & TS8 & TS9 & TS12 & TS15 & TS16 & TS20 & TS25 \\
\hline Minimum & 1 & 1 & 1 & 1 & 1 & 1 & 1 & 1 & 1 \\
\hline Maximum & 9 & 5 & 10 & 6 & 6 & 9 & 4 & 10 & 4 \\
\hline Mean & 3.82 & 1.89 & 4.40 & 2.77 & 2.26 & 4.02 & 1.89 & 4.33 & 1.62 \\
\hline SD & 1.68 & 0.98 & 2.25 & 1.35 & 1.24 & 1.94 & 1.03 & 2.21 & 0.73 \\
\hline
\end{tabular}


Table 2. Summary statistics of multiplicity of infection in samples of Theileria annulata from Portugal

\begin{tabular}{|c|c|c|c|c|c|c|}
\hline \multirow{2}{*}{ Country } & \multirow{2}{*}{ Region } & \multirow{2}{*}{$\mathbf{n}$} & \multicolumn{4}{|c|}{ Number of alleles per locus per isolate } \\
\hline & & & Mean & SD & Minimum & Maximum \\
\hline \multirow[t]{5}{*}{ Portugal } & & 90 & 3.06 & 0.75 & 1.11 & 4.50 \\
\hline & Évora & 40 & 3.16 & 0.78 & 1.11 & 4.33 \\
\hline & Portalegre & 29 & 2.83 & 0.72 & 1.38 & 4.22 \\
\hline & Ribatejo & 12 & 3.25 & 0.88 & 1.56 & 4.50 \\
\hline & Beja & 9 & 3.35 & 0.20 & 3.00 & 3.56 \\
\hline Tunisia & & 87 & 2.54 & 0.78 & 1.29 & 4.56 \\
\hline Turkey & & 218 & 3.12 & 1.13 & 1.00 & 5.78 \\
\hline
\end{tabular}


Table 3. Allelic variation in the Portuguese Theileria annulata population

1

2

\begin{tabular}{lcccccccccc}
\hline & TS5 & TS6 & TS8 & TS9 & TS12 & TS15 & TS16 & TS20 & TS25 & $\begin{array}{c}\text { All } \\
\text { loci }\end{array}$ \\
\hline Total number of alleles & 11 & 12 & 16 & 9 & 8 & 11 & 9 & 18 & 3 & 97 \\
Allelic richness & 12 & 12 & 16 & 9 & 9 & 12 & 10 & 18 & 4 & 102 \\
$\begin{array}{l}\text { Predominant allele size in bp } \\
\text { (frequency) }\end{array}$ & 300 & 390 & 183 & 357 & 228 & 235 & 348 & 223 & 218 & $(0.91)$ \\
Number of private alleles & $(0.46)$ & $(0.52)$ & $(0.27)$ & $(0.74)$ & $(0.76)$ & $(0.38)$ & $(0.41)$ & $(0.44)$ & $(0.91)$ & \\
\hline
\end{tabular}


Table 4. Gene diversity in Theileria annulata populations from Portugal, Tunisia and Turkey

1

2

\begin{tabular}{lcccccccccc}
\hline & TS5 & TS6 & TS8 & TS9 & TS12 & TS15 & TS16 & TS20 & TS25 & All loci \\
\hline Portugal & 0.753 & 0.657 & 0.865 & 0.439 & 0.408 & 0.801 & 0.749 & 0.785 & 0.167 & 0.625 \\
Tunisia & 0.827 & 0.946 & 0.946 & 0.960 & 0.956 & 0.829 & 0.898 & 0.885 & 0.708 & 0.884 \\
Turkey & 0.818 & 0.950 & 0.963 & 0.947 & 0.963 & 0.808 & 0.858 & 0.872 & 0.671 & 0.872 \\
\hline
\end{tabular}


Table 5. Genetic differentiation among Theileria annulata populations

\begin{tabular}{lll}
\hline & Portugal & Tunisia \\
\hline Portugal & - & \\
\hline Tunisia & $0.070^{*}$ & - \\
\hline Turkey & $0.048^{*}$ & $0.016^{*}$ \\
\hline${ }^{*}$ significant after Bonferroni corrections for multiple tests $(P<0.05)$
\end{tabular}


Table 6. Linkage equilibrium analyses in populations of Theileria annulata

\begin{tabular}{lclllll}
\hline Comparison & $\mathbf{n}$ & $\mathbf{I}_{\mathbf{A}}^{\mathbf{S}}$ & $\boldsymbol{V}_{\mathbf{D}}$ & L PARA & $\mathbf{L}_{\mathbf{M C}}$ & Linkage \\
\hline Portugal & 90 & 0.0272 & 2.0247 & 1.8521 & 1.8679 & LD \\
$\quad$ Évora & 40 & 0.0268 & 2.0677 & 1.9991 & 1.9727 & LD \\
$\quad$ Portalegre & 29 & 0.0368 & 2.0272 & 1.8874 & 1.9728 & LD \\
$\quad$ Ribatejo e Oeste & 12 & 0.0117 & 1.4762 & 1.8078 & 1.7531 & LE \\
$\quad$ Beja & 9 & 0.0164 & 1.4857 & 1.9837 & 1.9429 & LE \\
Tunisia & 87 & 0.0117 & 0.9534 & 0.9088 & 0.9128 & LD \\
Turkey & 218 & 0.0207 & 1.0671 & 0.9440 & 0.9495 & LD \\
Portugal, Tunisia and Turkey & 395 & 0.0538 & 1.1276 & 0.8032 & 0.8023 & LD \\
Portugal and Tunisia & 177 & 0.1677 & 2.3625 & 1.0480 & 1.0468 & LD \\
Portugal and Turkey & 308 & 0.0610 & 1.3447 & 0.9296 & 0.9254 & LD \\
Tunisia and Turkey & 305 & 0.0201 & 0.9136 & 0.8017 & 0,7993 & LD \\
\hline
\end{tabular}


Figure 1. Allele frequencies of TS5 in field populations of Theileria annulata. The frequency of the predominant allele in each sample was calculated for Portuguese $T$. annulata populations (grey) and compared with Tunisian (black) and Turkish (white) frequencies. 
Figure 2. Factorial correspondence analysis. Projection of genotypes from $(A)$ four regions of Portugal: Évora (black), Portalegre (light grey), Ribatejo (white) and Beja (dark grey). Projection of genotypes from Portugal, Tunisia and Turkey; (B) Portugal (white), Tunisia (grey) and Turkey (black). 


\section{References}

Aktas, M., Dumanli, N., Angin, M., 2004. Cattle infestation by Hyalomma ticks and prevalence of Theileria in Hyalomma species in the east of Turkey. Vet. Parasitol., 119, 1-8. doi:10.1016/j.vetpar.2003.10.013

Anderson, T.J., Haubold, B., Williams, J.T., Estrada-Franco, J.G., Richardson, L., Mollinedo, R., Bockarie, M., Mokili, J., Mharakurwa, S., French, N., Whitworth, J., Velez, I.D., Brockman, A.H., Nosten, F., Ferreira, M.U., Day, K.P., 2000. Microsatellite markers reveal a spectrum of population structures in the malaria parasite Plasmodium falciparum. Mol. Biol. Evol., 17, 1467-82.

Balloux, F., Lugon-Moulin, N., 2002. The estimation of population differentiation with microsatellite markers. Mol. Ecol., 11, 155-65.

Baptista, F.M., Nunes, T., 2007. Spatial analysis of cattle movement patterns in Portugal. Vet. Ital., 43, 611-9.

Belkhir, K., Borsa, P., Chikhi, L., Raufaste, N., Bonhomme, F., 1996-2004 GENETIX 4.05, logiciel sous Windows TM pour la génétique des populations. Laboratoire Génome, Populations, Interactions, CNRS UMR 5000, Université de Montpellier II, Montpellier (France).

Ben Miled, L., Dellagi, K., Bernardi, G., Melrose, T.R., Darghouth, M., Bouattour, A., Kinnaird, J., Shiels, B., Tait, A., Brown, C.G., 1994. Genomic and phenotypic diversity of Tunisian Theileria annulata isolates. Parasitology, 108, 51-60.

Branco, S., Orvalho, J., Leitão, A., Pereira, I., Malta, M., Mariano, I., Carvalho, T., Baptista, R., Shiels, B., Peleteiro, M.C., 2010. Fatal cases of Theileria annulata infection in calves in Portugal associated with neoplastic-like lymphoid cell proliferation. Journal of Veterinary Science, 11, 27-34. DOI: 10.4142/jvs.2010.11.1.27

Dobbelaere, D., and Heussler, V., 1999. Transformation of leukocytes by Theileria parva and T. annulata. Annual Review of Microbiology, 53, 1-42.

d'Oliveira, C., van der Weide, M., Habela, M.A., Jacquiet, P., Jongejan, F., 1995. Detection of Theileria annulata in blood samples of carrier cattle by PCR. J Clin Microbiol. 33, 2665-9.

Earl, D. A. and vonHoldt, B. M., 2012. STRUCTURE HARVESTER: a website and program for visualizing STRUCTURE output and implementing the Evanno method. Conservation Genetics Resources, 4, 359-361. 
Estrada-Peña, A., Santos-Silva, M.M., 2005. The distribution of ticks (Acari: Ixodidae) of domestic livestock in Portugal. Exp Appl Acarol. 36, 233-246. doi: 10.1007/s10493005-5107-9

Excoffier, L., Laval, G., Schneider, S., 2005. Arlequin ver. 3.0: An integrated software package for population genetics data analysis. Evolutionary Bioinformatics Online, 1, 47-50.

Garcia-Sanmartin, J., Nagore, D., Garcia-Perez, A. L., Juste, R. A., Hurtado, A., 2006. Molecular diagnosis of Theileria and Babesia species infecting cattle in Northern Spain using reverse line blot macroarrays. BMC Veterinary Research, 2, 16.

Gharbi, M., Hayouni, M.E., Sassi, L., Dridi, W., Darghouth, M.A., 2013. Hyalomma scupense (Acari, Ixodidae) in northeast Tunisia: seasonal population dynamics of nymphs and adults on field cattle. Parasite. 20, 281-283. doi: 10.1051/parasite/2013012.

Ghosh, S., Ray, D.D., Vanlahmuaka, Das G., Singh, N.K., Sharma, J.K., Azhahianambi, P., 2008. Progress in development of vaccine against Hyalomma anatolicum anatolicumIndian scenario. Vaccine. 19, 40-7. doi:10.1016/j.vaccine.2008.09.067

Gomes, J., Soares, R., Santos, M., Santos-Gomes, G., Botelho, A., Amaro, A., Inácio, J., 2013. Detection of Theileria and Babesia infections amongst asymptomatic cattle in Portugal. Ticks Tick Borne Dis., 4, 148-51. doi: 10.1016/j.ttbdis.2012.07.002.

Gubbels, J.M., de Vos, A.P., van der Weide, M., Viseras, J., Schouls, L.M., de Vries, E., Jongejan, F., 1999. Simultaneous detection of bovine Theileria and Babesia species by reverse line blot hybridization. J. Clin. Microbiol. 37, 1782-1789.

Gubbels, M.J., d'Oliveira, C., Jongejan, F., 2000. Development of an indirect Tams1 enzymelinked immunosorbent assay for diagnosis of Theileria annulata infection in cattle. Clinical and Diagnostic Laboratory Immunology, 7, 404-411.

Haubold, B., Hudson, R.R., 2000. LIAN 3.0: detecting linkage disequilibrium in multilocus data. Linkage Analysis. Bioinformatics. 16, 847-8.

Holm, S., 1979. A simple sequentially rejective multiple test procedure. Scandinavian Journal of Statistics. 6, 65-70.

Jakobsson, M., Rosenberg, N.A., 2007. CLUMPP: a cluster matching and permutation program for dealing with label switching and multimodality in analysis of population structure. Bioinformatics. 23, 1801-6. doi: 10.1093/bioinformatics/btm233

Jensen, J.L., Bohonak, A.J., Kelley, S.T., 2005. Isolation by distance, web service. BMC Genetics, 6, 13. doi: 10.1186/1471-2156-6-13 
Kalinowski, S.T., 2005. HP-Rare: a computer program for performing rarefaction on measures of allelic diversity. Molecular Ecology Notes 5, 187-189.

Mehlhorn, H., Schein, E., 1984. The piroplasms: life cycle and sexual stages. Adv. Parasitol. 23, 37-103.

Muleya, W., Namangala, B., Simuunza, M., Nakao, R., Inoue, N., Kimura, T., Ito, K., Sugimoto, C., Sawa, H., 2012. Population genetic analysis and sub-structuring of Theileria parva in the northern and eastern parts of Zambia. Parasit Vectors., 12, 255. doi:10.1186/1756-3305-5-255

Oura, C.A., Asiimwe, B.B., Weir, W., Lubega, G.W., Tait, A., 2005. Population genetic analysis and sub-structuring of Theileria parva in Uganda. Mol Biochem Parasitol., 140, 229-39. doi:10.1016/j.molbiopara.2004.12.015

Park. S.D.E., 2001. Trypanotolerance in West African cattle and the population genetics effects of selection. Ph.D thesis, University of Dublin, Dublin, Ireland.

Pritchard, J.K., Stephens, M., Donnelly, P., 2000. Inference of population structure using multilocus genotype data. Genetics, 155, 945-59.

Ross, A., Koepfli, C., Li, X., Schoepflin, S., Siba, P., Mueller, I., Felger, I., Smith, T., 2012. Estimating the Numbers of Malaria Infections in Blood Samples Using HighResolution Genotyping Data. PLoS ONE. 7 (8). doi: 10.1371/journal.pone.0042496

Santos, M., Soares, R., Costa, P., Amaro, A., Inácio, J., Gomes, J., 2013. Revisiting the Tams1-encoding gene as a species-specific target for the molecular detection of Theileria annulata in bovine blood samples. Ticks Tick Borne Dis., 4, 72-7. doi: 10.1016/j.ttbdis.2012.07.006

Schnittger, L., Katzer, F., Biermann, R., Shayan, P., Boguslawski, K., McKellar, S., Beyer, D., Shiels, B.R., Ahmed, J.S., 2002. Characterization of a polymorphic Theileria annulata surface protein (TaSP) closely related to PIM of Theileria parva: implications for use in diagnostic tests and subunit vaccines. Mol. Biochem. Parasitol. 120, 247-256.

Shiels, B., McDougall, C., Tait, A., Brown, C.G., 1986. Antigenic diversity of Theileria annulata macroschizonts. Vet Parasitol., 21, 1-10.

Shiels, B., Kinnaird, J., McKellar, S., Dickson, J., Miled, L.B., Melrose, R., Brown, D., Tait, A., 1992. Disruption of synchrony between parasite growth and host cell division is a determinant of differentiation to the merozoite in Theileria annulata. J. Cell Sci. 101, 99-107. 
Silva, M. G., Marques, P. X., Oliva, A., 2010. Detection of Babesia and Theileria species infection in cattle from Portugal using a reverse line blotting method. Vet. Parasitol., 174, 199-205. doi:10.1016/j.vetpar.2010.08.038

Weir, B.S., Cockerham, C.C., 1984. Estimating F-Statistics for the Analysis of Population Structure. Evolution, 38, 1358-1370.

Weir, W., Ben Miled, L., Karagenc, T., Katzer, F., Darghouth, M.A., Shiels, B.R., Tait, A., 2007. Genetic exchange and sub-structuring in Theileria annulata populations. Mol. Biochem. Parasitol. 154, 170-180. doi:10.1016/j.molbiopara.2007.04.015

Weir, W., Karagenc, T., Baird, M., Tait, A., Shiels, B. R., 2010. Evolution and diversity of secretome genes in the apicomplexan parasite Theileria annulata. BMC Genomics, 11, 42. doi: 10.1186/1471-2164-11-42.

Weir, W., Karagenc, T., Gharbi, M., Simuunza, M., Aypak, S., Aysul, N., Darghouth, M.A., Shiels, B., Tait, A., 2011. Population diversity and multiplicity of infection in Theileria annulata. International Journal for Parasitology, 41, 193-203. doi: 10.1016/j.jpara.2010.08.004. 


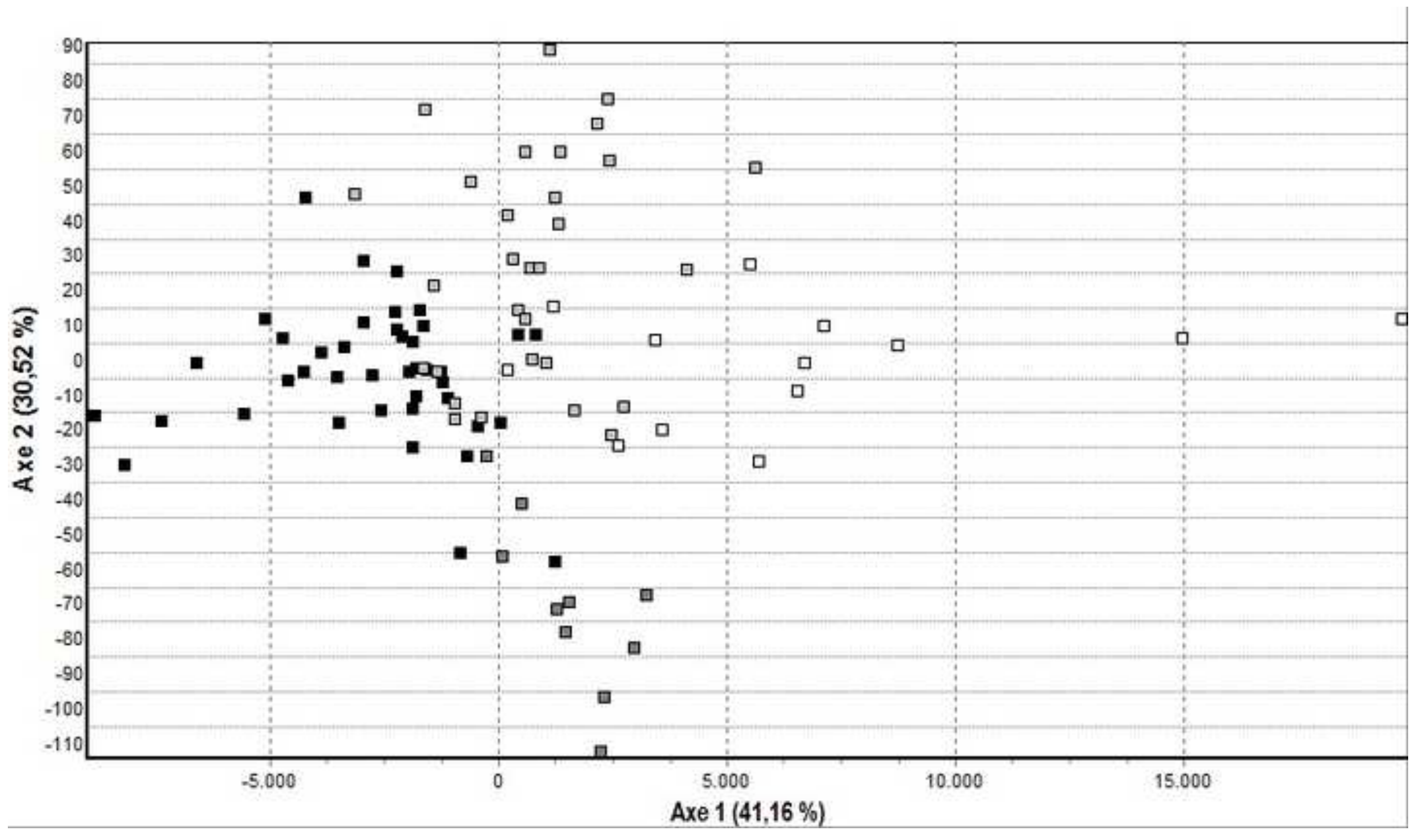




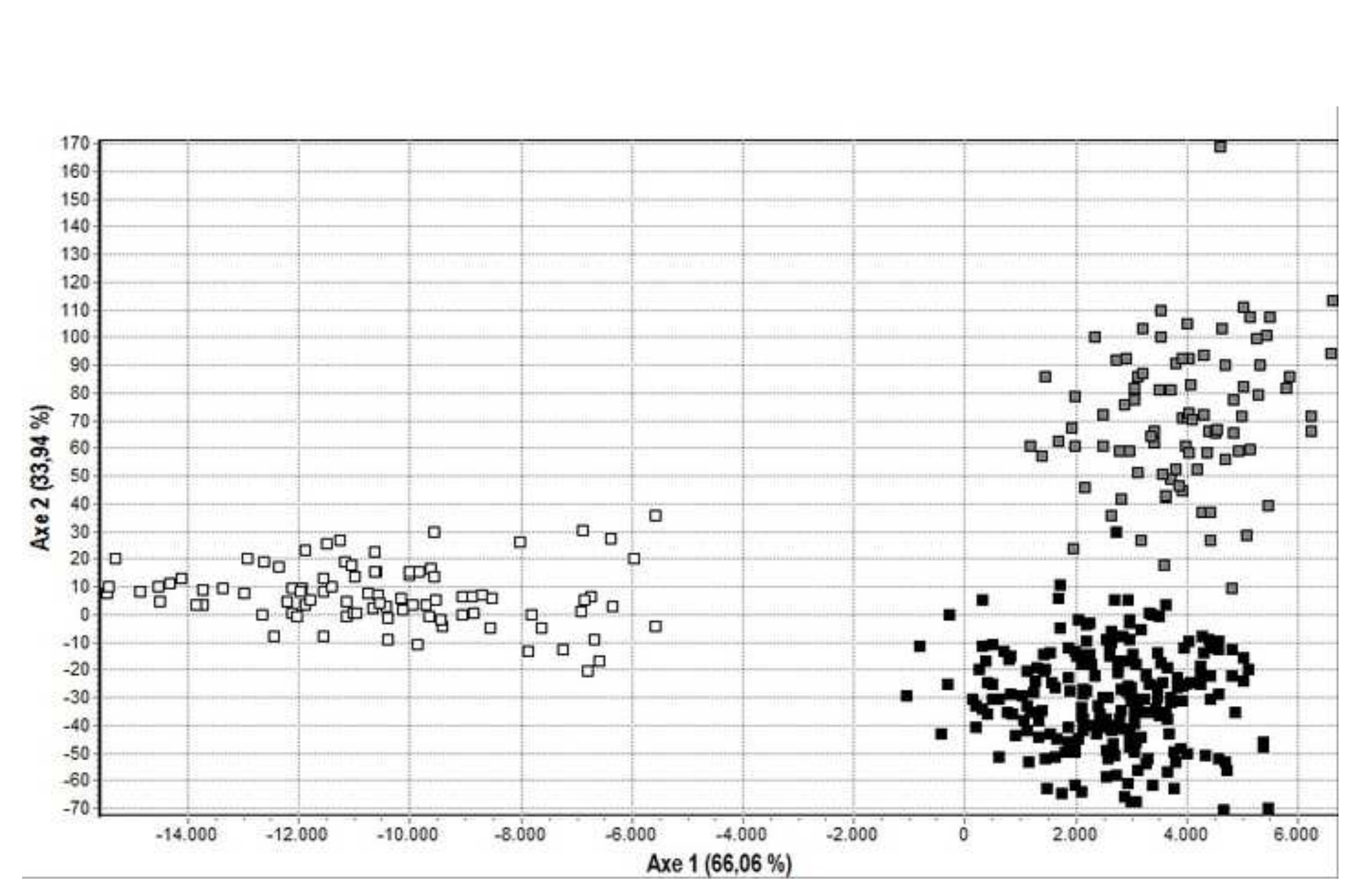

\section{Figure2B}

.

.
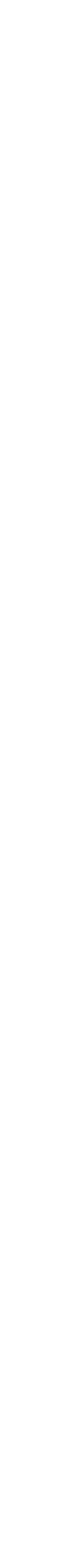